\title{
Automated Interventricular Septum Thickness Measurement from B-Mode Echocardiograms
}

\author{
Navneeth Subramanian ${ }^{1}$, Dirk Padfield ${ }^{2}$, Sheshadri Thiruvenkadam ${ }^{1}$, \\ Anand Narasimhamurthy ${ }^{1}$, and Sigmund Frigstad ${ }^{3}$ \\ 1 GE Global Research, Whitefield Road, Bangalore, India \\ navneeth.s@ge.com \\ ${ }^{2}$ GE Global Research, One Research Circle, Niskayuna, NY \\ ${ }^{3}$ GE Vingmed Ultrasound, Trondheim, Norway
}

\begin{abstract}
In this work, we address the problem of automated measurement of the interventricular septum thickness, one of the key parameters in cardiology, from B-mode echocardiograms. The problem is challenging due to high levels of noise, multi modal intensity, weak contrast due to near field haze, and non rigid motion of the septum across frames. We introduce a complete system for automated measurement of septum thickness from B-mode echocardiograms incorporating three main components: a 1D curve evolution algorithm using region statistics for segmenting the septum, a motion clustering method to locate the mitral valve, and a robust method to calculate the septum width from these inputs in accordance with medical standards. Our method effectively handles the challenges of such measurements and runs in near real time. Results on 57 patient recordings showed excellent agreement of the automated measurements with expert manual measurements.
\end{abstract}

\section{Introduction}

Interventricular septum thickness (or diameter) (IVSd) is one of the key parameters in cardiology since it is, along with LV size, one of the main indicators of cardiac hypertrophy. It can be used as a screening parameter for septal hypertrophy and has also shown a correlation to $24 \mathrm{~h}$ ambulatory blood pressure. Unfortunately, the manual measurement of the septum thickness on echocardiograms suffers from large inter- and intra-observer variability based on the experience of the user. Further variability results from different acquisitions since it is not easy to pick the right plane to scan, and this challenge is compounded by misalignment between available acoustic windows for imaging and the patient's heart. These issues can lead to undesirable variations in the diagnosis, resulting in missed detection of subjects and unnecessary further testing of healthy patients. Automating this measurement can avoid this variability and can save physicians time and effort.

Close observation reveals that the IVSd measurement is a complex cognitive task performed by the cardiologist. An end-to-end IVSd measurement in accordance with the American Society of Echocardiography (ASE) guidelines [1]

T. Jiang et al. (Eds.): MICCAI 2010, Part I, LNCS 6361, pp. 510-517, 2010.

(C) Springer-Verlag Berlin Heidelberg 2010 
involves segmentation of the septum, identification of the tip of the mitral valve, and measurement of the thickness orthogonal to the centerline of the septum. The aforementioned procedure must be repeated for all frames of a multi-frame recording, and the septum thickness is reported on the frame corresponding to end-diastole.

Our contribution is a solution for end-to-end automation of the complex septum measurement workflow on PLAX B-mode echocardiograms. Our method incorporates three main components: a 1D curve evolution algorithm for segmenting the septum on each frame, a motion-clustering based method to localize the mitral valve, and a robust method to calculate the septum width from these inputs in accordance with medical standards. We know of no previous attempt at end-to-end septum measurement automation. For this reason, we compare against previously developed approaches for septum segmentation and identification of the mitral valve, individually.

Any approach to segment the septum from ultrasound images faces several challenges. Firstly, the intensity in and around the septum region is multi modal, hence purely intensity and histogram based schemes are challenged. For example, the approach proposed in [2] uses multi-level thresholding for measurement on a single instantaneous frame. This method, however, has an inherent difficulty on tough clinical cases where near-field haze is encountered. Secondly, the speckle noise inherent in ultrasound images makes edge-based methods unreliable. These methods are sensitive to initialization and are plagued by the near-field haze which leads to low contrast at the upper part of the septum. For any approach to segment the septum across several frames reliably, robustness to noise and initialization is needed. Region based active contour [AC] approaches have typically offered robustness to curve placement and noise, but methods using global statistics [3] are not ideal for segmenting objects from multi-modal data, as in our case. Recent works [415] have addressed this issue by locally estimating statistics around each pixel, although at a higher computational cost. There are also efforts that introduce prior shape information into segmentation schemes based on [AC] [6 7], and these methods have been reasonably successful in handling low contrast and boundary gaps, but, to be effective, they need to be carefully trained to acquire a rich description of shape statistics. However, the shape of the septum varies vastly across patients and with view angles, and undergoes large non rigid motion across frames. These factors make it difficult to build a shape atlas of the septum to use within a segmentation approach.

We propose a local region based variational energy to solve the segmentation problem. One main difference as compared to usual $2 \mathrm{D}[\mathrm{AC}]$ methods is in our representation of the septum. As a result of insights gained from clinical data, we represent the septum using two 1D profiles which correspond to the top and bottom septum boundaries respectively. This representation allows capture of regional statistics in and around the septum, and model interractions between the boundaries (e.g. width) of the septum, and is computationally easier. With the above terms, our segmentation energy was able to effectively deal with the data challenges and viable for real time implementation. 


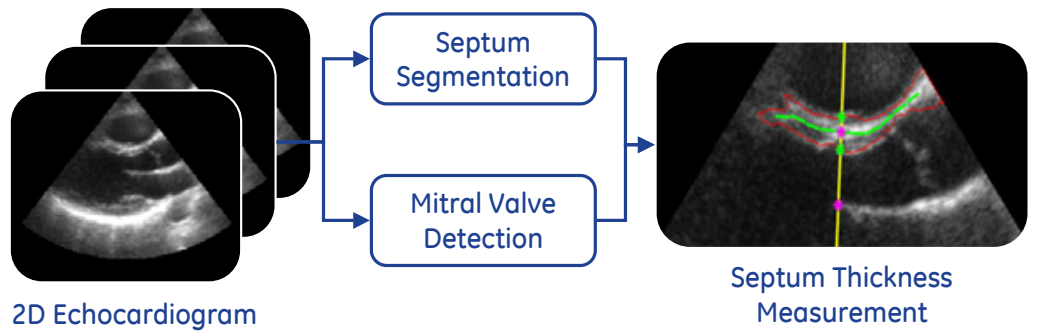

Fig. 1. Fully automatic IVSd measurement: The right figure shows the segmented septum in red, the detected mitral valve tip as a purple star, the fit of piecewise lines to the septum medial axis as green lines, the computation of the orthogonal line in yellow, and calculation of the points used to define septum thickness as green stars

Next, a critical step for defining the measurement line of the septum thickness is the identification of the mitral valve leaflet. Martin et al. 8] propose a semi-automatic method where a rough segmentation is first obtained and is then refined using active contours with dynamic program minimization. However, as outlined by the authors, the approach is sensitive to the manual initialization of the contours at the muscle-leaflet junction, precluding its application in automated measurement. We propose a simple method, motivated by the observation that valve leaflets are the fastest moving cardiac structures. The novelty of our method is that it is completely unsupervised without the need for prior training or initialization as compared to previous approaches.

Finally, given the segmented septum and the location of the mitral valve tip, the thickness measurement is given by the length of the septum region along the line that is orthogonal to the center line of the septum region and passing through the mitral valve tip. Our end to end method was validated on a large variety of patient recordings, many with challenging image quality, and provided reproducible measurement that follow the guidelines of the ASE. The automated measurements closely correspond with manual measurements generated by cardiologists from two separate institutions.

\section{Methods}

Our approach divides the problem into three steps that can be solved independently: septum segmentation, mitral valve tip detection, and septum thickness measurement as shown in Figure 1 .

\subsection{Septum Segmentation}

As mentioned in the introduction, rather than utilize 2D contour evolution approaches, we formulate our search space over pairs of smooth $1 \mathrm{D}$ profiles. This representation enables easy access to regional statistics in and around the septum, and model interactions between the top and bottom septum boundaries, 
in our case, to broadly constrain the width of the segmented septum. The width term is crucial for effective segmentation under weak contrast at the top part of the septum due to near field haze. Also from a computational standpoint, our 1D-profile based formulation works over a smaller set of minimization arguments than regular $[\mathrm{AC}]$ representations. Thus the resulting segmentation is very fast making it feasible for real time use.

Given an ultrasound image $I: \Omega \rightarrow R, \Omega=[a, b] \times[c, d]$, the goal is to search for two smooth 1D functions $f, g:[a, b] \rightarrow[c, d]$, whose profiles represent the top and bottom parts of the septum. We assume that close to the septum boundary, the image intensity is piecewise constant. Hence we seek an $f$ and $g$ that separate local neighborhoods into three regions (see Figure 2), each of which we model with Gaussian statistics. We denote the septum region, the region between the $1 \mathrm{D}$ profiles of $f$ and $g$, as $R^{s}$. We denote the neighborhood region above the septum as $R^{a}$, i.e. between profiles of $g$ and $g+\Delta$, where $\Delta$ is some pre-defined interval. Similarly, the neighborhood below the septum is $R^{b}$, between profiles of $f-\Delta$ and $f$. Rather than represent the entire septum region by one distribution, we divide $\Omega$ into $K$ disjoint rectangles, $\Omega=\bigcup_{i=1}^{K} B_{i}$, $B_{i}=\left(a_{i}, b_{i}\right) \times(c, d)$, which yields multiple, more accurate distributions. In this way, the image intensity is expected to be Gaussian in the regions $R^{s} \cap B_{i}, R^{a} \cap B_{i}$, and $R^{b} \cap B_{i}$. Under this construction, $\mu^{a}=\left[\mu_{1}^{a}, \mu_{2}^{a}, . ., \mu_{K}^{a}\right], \sigma^{a}=\left[\sigma_{1}^{a}, \sigma_{2}^{a}, . ., \sigma_{K}^{a}\right]$ gives the set of distributions in each of the regions $R^{a} \cap B_{i}$, similarly $\mu^{b}, \sigma^{b}$ gives the distribution in each $R^{b} \cap B_{i}$, and $\mu^{s}, \sigma^{s}$ gives the distribution in each $R^{s} \cap B_{i}$. To find the septum region, we minimize the following energy over the space of smooth 1D functions $f, g:[a, b] \rightarrow[c, d]$, with distributions $\mu^{a}, \sigma^{a}, \mu^{s}, \sigma^{s}, \mu^{b}, \sigma^{b}$ :

$$
\begin{aligned}
& E\left(f, g, \mu^{a}, \sigma^{a}, \mu^{s}, \sigma^{s}, \mu^{b}, \sigma^{b}\right)=\sum_{i=1}^{K}\left[\int_{a_{i}}^{b_{i}} \int_{f}^{g}\left(\frac{\left(I-\mu_{i}^{s}\right)^{2}}{\left(\sigma_{i}^{s}\right)^{2}}+\ln \left(\sigma_{i}^{s}\right)\right)\right. \\
& \left.+\int_{a_{i}}^{b_{i}} \int_{f-\Delta}^{f}\left(\frac{\left(I-\mu_{i}^{b}\right)^{2}}{\left(\sigma_{i}^{b}\right)^{2}}+\ln \left(\sigma_{i}^{b}\right)\right)+\int_{a_{i}}^{b_{i}} \int_{g}^{g+\Delta}\left(\frac{\left(I-\mu_{i}^{a}\right)^{2}}{\left(\sigma_{i}^{a}\right)^{2}}+\ln \left(\sigma_{i}^{a}\right)\right)\right] \\
& +\lambda_{\text {smooth }} \int_{a}^{b}\left(\sqrt{1+\left(f^{\prime}\right)^{2}}+\sqrt{1+\left(g^{\prime}\right)^{2}}\right)+\lambda_{\text {width }} \int_{a}^{b}(f+w-g)^{2}
\end{aligned}
$$

The data term drives $f, g$ to take values such that the intensity distribution in each of $R^{s} \cap B_{i}, R^{a} \cap B_{i}$, and $R^{b} \cap B_{i}$ are Gaussian, given by $\mu^{a}, \sigma^{a}, \mu^{s}, \sigma^{s}, \mu^{b}, \sigma^{b}$. The smoothness terms for $f$ and $g$ are governed by parameter $\lambda_{\text {smooth }}$. The last term constrains the width of the region $R^{s}$ to be close to the expected septum width $(w)$ and is balanced by $\lambda_{w i d t h}$. To minimize $E$, we use the Euler Lagrange equations of Equation 11 to iteratively solve for $f, g$, and $\mu^{a}, \sigma^{a}, \mu^{s}, \sigma^{s}, \mu^{b}, \sigma^{b}$, using an explicit finite difference scheme and given an initial guess $f_{0}, g_{0}$. Figure 2 illustrates our segmentation evolution on a sample image.

\subsection{Mitral Valve Detection}

Locating the mitral valve leaflet is critical to septum measurement since clinical guidelines require measurement to be done at the level of the mitral valve in 

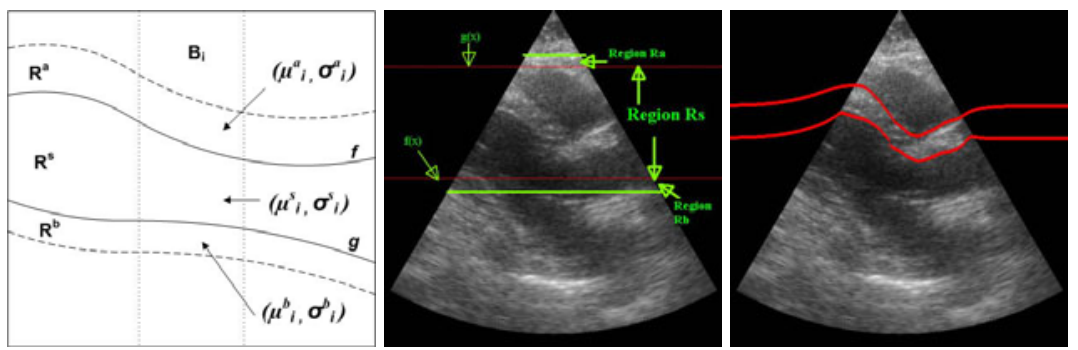

Fig. 2. $f, g$ represent the lower and upper boundaries of the septum, respectively. The initialization of the functions (middle), and final segmentation of the septum (right). Regional statistics in the septum $\left(R^{s}\right)$, above the septum $\left(R^{a}\right)$, and below the septum $\left(R^{b}\right)$ are used to drive evolution of the functions $f, g$.

the end-diastole frame. This task is challenging since the mitral valve is a fast moving and thin structure, but we introduce a method to locate the valve leaflet tip that leverages this characteristic. The main advantages of our method is that it is fast and is completely unsupervised without the need for prior training or initialization. It consists of computing a motion map, identifying candidate locations corresponding to high motion, and computing a representative location corresponding to the anterior tip of the mitral valve. The motion map is computed via frame differencing considering a window of several frames. It is necessary to consider more than one set of frame differences because the mitral valve leaflet is not clearly visible in some frames so that only one frame difference may not yield many locations corresponding to the valve leaflet. Next, pixels corresponding to high motion are identified as candidate locations, and these are grouped into clusters using a $K$-means clustering algorithm. The cluster center is then representative of the valve location. In our case, we are interested only in the mitral valve, and the cluster center corresponds with the mitral valve tip. The algorithm steps are illustrated in Figure 3 .

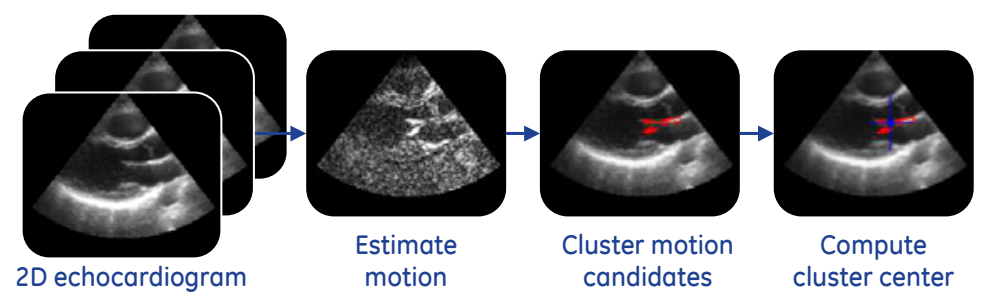

Fig. 3. Block diagram of the method for localizing the mitral valve leaflet 


\subsection{Septum Thickness Measurement}

The manual measurement protocol of the ASE specifies that the measurement line should be drawn orthogonal to the center line of the septum region and passing through the mitral valve tip. We embed this requirement directly into our septum thickness algorithm, which is demonstrated in Figure 1, To represent the center line, our method first calculates the medial axis of the septum region. To make the algorithm computationally efficient, we then divide the medial axis into $K$ regions (for example $K=10$ works well) and fit line segments to each of these regions using linear regression. These $K$ line segments are shown as green lines in Figure 1, Given the $K$ line segments, we find the line through the mitral valve tip $p_{m}$ with the smallest distance to each line segment $\overline{p_{s} p_{e}}$ (where $p_{s}$ and $p_{e}$ are the start and end point of the line, respectively). Each of these lines is orthogonal to its corresponding fit line segment by definition, and they intersect at point $p_{i}$ that is found as $p_{i}=p_{s}+t *\left(p_{e}-p_{s}\right)$ where $t=-\frac{\left(p_{s}-p_{m}\right) \cdot\left(p_{s}-p_{e}\right)}{\left\|p_{e}-p_{s}\right\|^{2}}$ is the distance to $p_{i}$ from $p_{s}$. Given the intersection point $p_{i}$ and the mitral valve tip location $p_{m}$, the Euclidean distance between these two points is calculated. This method is repeated for all of the line segments fit to the septum region, and the line with the shortest distance is chosen for the septum thickness calculation. The septum thickness is found as the distance between the two points where this line intersects the septum segmentation mask.

\section{Results}

We evaluated our framework on two databases of B-mode parasternal long axis (PLAX) recordings, representing a total of 57 different patients. Each recording has several cardiac cycles ( $\approx 3$ cycles/recording).The datasets were acquired on GE Vivid series ultrasound scanners at clinical sites in two countries. Two cardiologists independently performed manual measurements of IVSd on all datasets. Both cardiologists used the ECG to identify the end-diastole frame, on which the septum thickness was measured using calipers in B-mode. One of the cardiologists also scored the images as good, moderate, or poor based on the image quality and difficulty in imaging the patient.

The performance of the automated measurement algorithm was evaluated by comparing its measurement value on the selected frame with the cardiologists' measurements as shown in Figure 4. We report a mean error of $0.88 \pm 0.96 \mathrm{~mm}$ $(\min =0.0 \& \max =5.0 \mathrm{~mm})$ with respect to cardiologist 1 , and an error of 1.17 $\pm 0.92 \mathrm{~mm}(\min =0.0 \& \max =3.6 \mathrm{~mm})$ with respect to cardiologist 2 . The interobserver weighted kappa statistic was 0.58 . The weighted kappa statistic for our algorithm (0.56 vs. cardiologist $1,0.49$ vs. cardiologist 2 ) compares favourably with the inter-observer value. Figure 5 shows results on example good, moderate and poor quality recordings as defined by the cardiologists. Note the robustness of the algorithm to near-field haze and poor contrast.

In a clinical application, one uses the septum measurement to classify patients as normal, mildly abnormal, or hypertrophic.Standard recommendations on septum thickness for each of these categories are separated by approximately $2 \mathrm{~mm}$. 

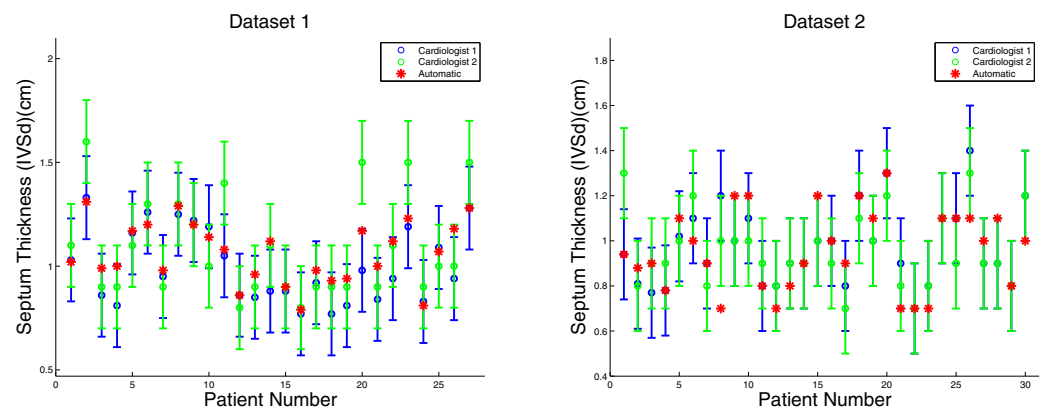

Fig. 4. Comparison of automatic versus manual measurements for two datasets representing a total of 57 patient recordings. The plot on the left shows results for 27 cases, and that on the right shows 30 cases. The blue and green circles are the manual measurements from two different expert cardiologists, and each circle shows the $\pm 2 \mathrm{~mm}$ range considered acceptable by the ASE standards. The red stars show the measurements from the automated method, which, in most cases, are within the range of the manual measurements.
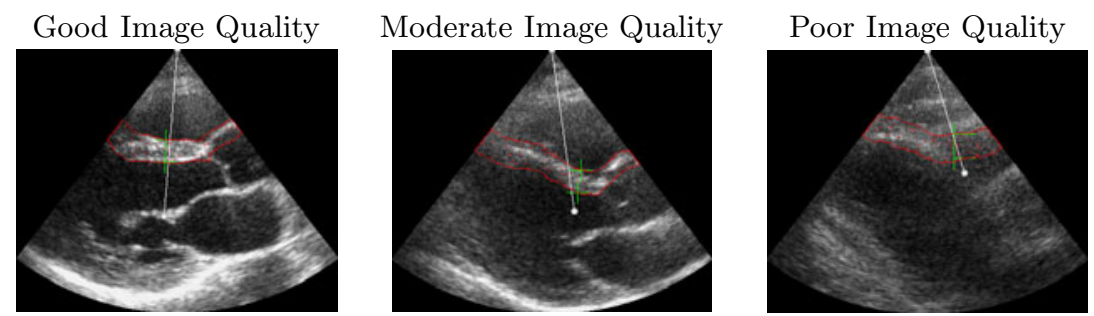

Patient 3: 0.86, 0.90, 0.99
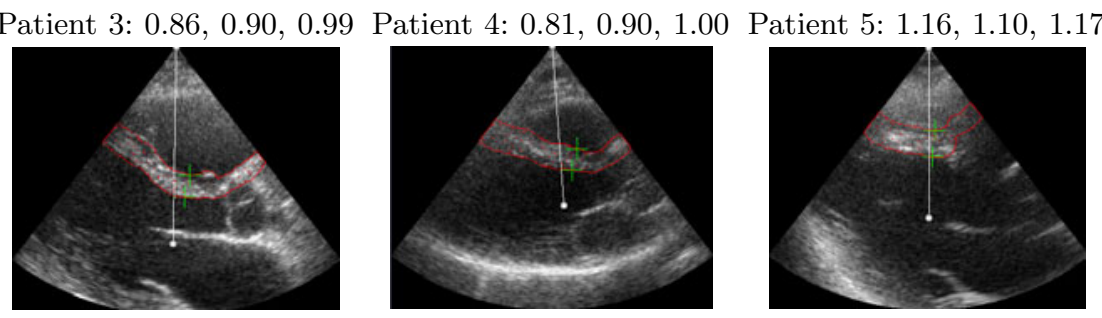

Patient 17: 0.92, 0.90, 0.98 Patient 7: 0.95, 0.90, 0.98 Patient 10: 1.19, 1.00, 1.14

Fig. 5. Visual comparison of example measurement results from Dataset 1. The three columns represent good, moderate, and poor image acquisitions, respectively, as classified by expert cardiologists. The text under each figure lists the patient number (see the left plot of Figure 4) followed by the thickness measurement by cardiologist 1, cardiologist 2 , and the automatic system, respectively. The red outline defines the automatic septum segmentation, and the green "+" signs indicate the locations of the septum width measurement. The white line is given as a reference to show the line connecting the tip of the probe with the detected mitral valve tip location. 
Hence, defining the success criterion as the automated approach being within $2 \mathrm{~mm}$ of the cardiologists measurement is clinically acceptable. Using this criterion, the success ratio of the automatic septum measurement solution (within $\pm 2 \mathrm{~mm}$ of the expert measurement) was found to be $89 \%$ (51/57 cases) with respect to cardiologist 1 and $82 \%$ (47/57 cases) with respect to cardiologist 2 . Our method runs in near real-time, with an average computation time of 0.1 sec/frame on a $2.6 \mathrm{GHZ} \mathrm{PC}$ with $2 \mathrm{~GB}$ RAM.

\section{Conclusions}

We have developed an automatic approach for septum thickness measurement consisting of septum segmentation using 1D curve evolutions based on regional statistics, mitral valve tip detection, and measurement line computation. The framework and its steps were developed in accordance with the clinical standards of the ASE, and they run in near real time. Our framework was validated on 57 $\mathrm{B}$-mode PLAX recordings and was within the $\pm 2 \mathrm{~mm}$ guidelines specified by the ASE in an average of $85 \%$ of the cases. This automated measurement solution compares favorably with manual measurements by expert cardiologists and is attractive for clinical application.

Acknowledgements. The authors would like to thank Feng Lin, Kajoli Krishnan and Kai Thomenius for valuable insight and discussions.

\section{References}

1. Lang, R., et al.: Recommendations for chamber quantification: a report from the American Society of Echocardiographys Guidelines and Standards Committee. Journal of the American Society of Echocardiography 18(12), 1440-1463 (2005)

2. Moladoust, H., et al.: Estimation of septal wall thickness by processing sequential echocardiographic images. Iranian Cardiovascular Research Journal 3(1) (2009)

3. Chan, T.F., Vese, L.A.: Active contours without edges. IEEE Trans. Image Processing 10, 266-277 (2001)

4. Guo, W., Chen, Y.: Using non-parametric kernel to segment and smooth images simultaneously. In: ICIP, pp. 217-220 (2006)

5. Lankton, S., Tannenbaum, A.: Localizing region-based active contours. IEEE Trans. Image Processing 17, 2029-2039 (2008)

6. Leventon, M., Grimson, W.L., Faugeras, O.: Statistical shape influence in geodesic active contours. In: CVPR, vol. 1, pp. 316-323 (2000)

7. Chen, Y., Thiruvenkadam, S., et al.: On the incorporation of shape priors into geometric active contours. In: IEEE VLSM, pp. 145-152 (2001)

8. Martin, S., Daanen, V., et al.: Tracking of the mitral valve leaflet in echocardiography images. In: IEEE Intl. Symp. on Biomedical Imaging, ISBI (2006) 